\title{
Defects in colloidal crystal
}

\author{
R KESAVAMOORTHY* and C BABU RAO \\ Metallurgy and Materials Group, Indira Gandhi Centre for Atomic Research, Kalpakkam \\ 603102 , India
}

\begin{abstract}
Colloidal crystal of polystyrene spheres of $0.53 \mu \mathrm{m}$ diameter dispersed in water is prepared in a quartz container. Optical microscope, video camera and image processor are used to investigate the defects, their dynamics and the effect of shear in this colloidal crystal. Six layers of the crystal from the surface of the container are imaged sequentially, one after another, and the crystal is reconstructed. FCC structure with (111) planes parallel to the container surface is stable at high volume fraction whereas, at low volume fraction $(100)$ planes also coexist. Vacancies, dislocations, grain boundaries, voids and particle aggregates are identified. The vibration of aggregates is sluggish as compared to that of a single particle. The nearest layer of particles to the container surface is better ordered than those in the interior layers. The container wall provides a stabilizing potential to the nearby layers of particles. The 2-D crystalline-like characteristics of the nearby layers are probed. The colloidal crystal is shear-melted by shaking the container. On resting it, the crystal-flow slows down. The flow is constrained to be parallel to the container surface and occurs along the layer in quantum jumps. During the flow, both (111) and (100) planes coexist in the layer. Flow at low rates anneals the defects created by the shear-melting.
\end{abstract}

Keywords. Colloidal crystal; defects; optical microscopy; 2-D crystal; shearing; crystal-flow.

\section{Introduction}

The submicron sized spherical colloidal polystyrene particles acquire negative charge on its surface when dispersed in water due to the dissociation of acidic end groups in water. They interact among themselves via screened Coulomb pair potential. On varying one or more parameters like the ionic impurity concentration in the suspension, the temperature, the dielectric constant of the suspension, the size and the charge of the particle and the particle concentration, the range and the strength of the interaction can be varied and the colloidal particles exhibit various order like crystal, glass, liquid and gas (Clark 1979; Linsdsay and Chaikin 1982; Piranski 1983; Hirtzel and Rajagopalan 1985; Ise 1986; Kesavamoorthy et al 1988; Robbins et al 1988; Russel et al 1988; Rundquist et al 1991). These colloidal dispersions and their crystalline order are extremely delicate and hence are termed as soft condensed matter. The phases of colloidal suspensions have a lot of similarities with atomic systems. The elastic constants and the thermodynamic parameters of colloidal suspensions and the atomic systems show remarkable scaling with the particle concentration (Linsdsay and Chaikin 1982; Hirtzel and Rajagopalan 1985). Study of the structural order in colloidal suspensions is very simple due to their large lattice parameters and is rich in information. Colloidal suspensions are treated as model systems to probe and predict the material behaviour under extreme conditions which are otherwise not practical in atomic system.

Optical microscopy has been used extensively to investigate the ordering in colloidal suspensions (Piranski 1983; Ise 1985; Ito et al 1988; Murray et al 1990). Ise (1985) and Ito et al (1988) have shown the coexistence of the colloidal crystal and liquid, the dynamic character of the boundary between them and the thermal motion of the particles at various temperatures. Pansu et al (1983) studied thin layers of colloidal crystals

\footnotetext{
*Author for correspondence
} 
confined between two glass plates. The glass wall provides a stabilizing potential to the particles near to it due to the induced image charge effect (Kesavamoorthy et al 1992). A single layer of colloidal particles on the air-water interface (Amstrong et al 1989; Kesavamoorthy et al 1993) or that confined in water between two glass plates (Piranski 1980) has been investigated for its 2-D melting and instability. The effect of shear on colloidal crystal has been investigated by optical diffraction (Clark et al 1979).

In this work, we report the direct microscope observation of ordering in aqueous suspension of polystyrene particles of diameter $0.53 \mu \mathrm{m}$ with volume percent ranging from 0.05 to 2 . We reconstruct 3-D colloidal crystal from the acquired images of 6 layers of particles near the container wall using image processor. We discuss the observation of defects, grains and the 2-D characteristics of the layer near to the wall in these colloidal crystals. We shear the crystal and observe the behaviour of the particles in the first layer.

\section{Experimental}

Monodisperse aqueous suspension of polystyrene spheres of $0.53 \mu \mathrm{m}$ diameter with 2.5 vol. \% (from Polysciences Inc, USA) was gently ultrasonicated to disperse the loosely bound aggregates and passed through a column of mixed-bed ion-exchange (cation, Ambercep 252; anion, Ambercep 900) repeatedly to purify the suspension. After it reached a conductivity of about $0.5 \mu \mathrm{S} \mathrm{cm}^{-1}$, it was sealed in the observation cells with various vol. $\%$ ranging from 0.05 to 2 by dilution. The cell was a cylindrical quartz tube of $1 \mathrm{~cm}$ diameter and $1 \mathrm{~cm}$ height with the top and bottom surfaces nicely polished and closed with $133 \mu \mathrm{m}$ thick glass plates using epoxy resin (araldite). The mixed-bed ion-exchange resin was taken into the cell to fill nearly three-quarters of its volume and the suspension filled the rest of the cell without air bubble. The resins settled down in the cell. Deionization equilibrium was reached in about a week (Kesavamoorthy and Arora 1985) and then the observation was started. The region of observation was close to the top glass plate and was away from the resin and other walls of the cell atleast by $2 \mathrm{~mm}$. Optical reflection microscope (Zeiss, Germany) having an oil-immersion objective $(\times 100)$ fitted with video camera (Andrex, Denmark) and an image processor (Andrex, Denmark) was used. The microscope-light intensity was kept at the minimum necessary level in order to avoid the local heating of the colloidal crystal. Focussing at different depths in the suspension was achieved by calibrated Z-movement of the microscope sample platform. The interlayer separation in the colloidal crystal was large enough as compared to the field of depth $(0.5 \mu \mathrm{m})$, such that observation/imaging was confined to a single layer. Image of six layers of particles were grabbed one after another using the Z-movement of the microscope and stored successively. The Z-coordinate for each layer was then assigned and 3-D crystal structures were reconstructed using the image processor. The observations were started 30 min after resting the cell in the microscope platform in order to reach the gravitational equilibrium (Kesavamoorthy and Arora 1985; Kesavamoorthy 1990). However, in order to investigate the effect of shear on colloidal crystal layer, the observation was started immediately after resting the shaken-cell in the sample platform.

\section{Results and discussions}

Figure 1 shows a typical micrograph of a layer of the colloidal crystal. Vacancies, dislocations and aggregates of particles are seen in the figure. It shows a hexagonal 
order of particle in the layer. After reconstructing the 3-D colloidal crystal, this layer was identified as (111) plane of FCC.

\subsection{Residual stress}

The distribution of the interparticle spacing, $d$, is obtained from 200 pairs of particles in figure 1 and is shown in figure 2. The distribution along [100] direction is shown in figure $2 a$ while that along $[1, \sqrt{3}, 0]$ and $[1,-\sqrt{3}, 0]$ is shown in figure $2 b$. This figure indicates that the particles along [100] direction are compressed as compared to those along the other two directions. This might be due to the residual stress present in the crystal after shaking. The distribution of $d$ taken after a day was the same in all the three directions. The residual stress might have been relieved in a day. Figure 2c shows the distribution of $d$ in the second layer along all directions within $1 \mathrm{~h}$ after shaking. This distribution is broad. This figure indicates that the residual stress is not present in the second layer. The weighted mean of the $d$ in the first layer and the second layer is the same $(2.44 \mu \mathrm{m}$ for the vol. $\%$ of 0.73$)$. The fraction of six coordinated particles in the first layer was about 0.8 and that in the second layer 0.6 . The number of defects in a layer increases from the first layer towards the interior. All these observations imply that the first layer is ordered better than other layers (Kesavamoorthy et al 1989). The first layer particles are held in position by a steeper potential well as compared to other layer particles. Hence, annealing of residual stress took longer time in the first layer.

\subsection{2-D characteristics of the layers}

During the process of crystallization, it was observed that the first layer near the glass surface orders first, then the second layer and so on. During the melting of the crystal by

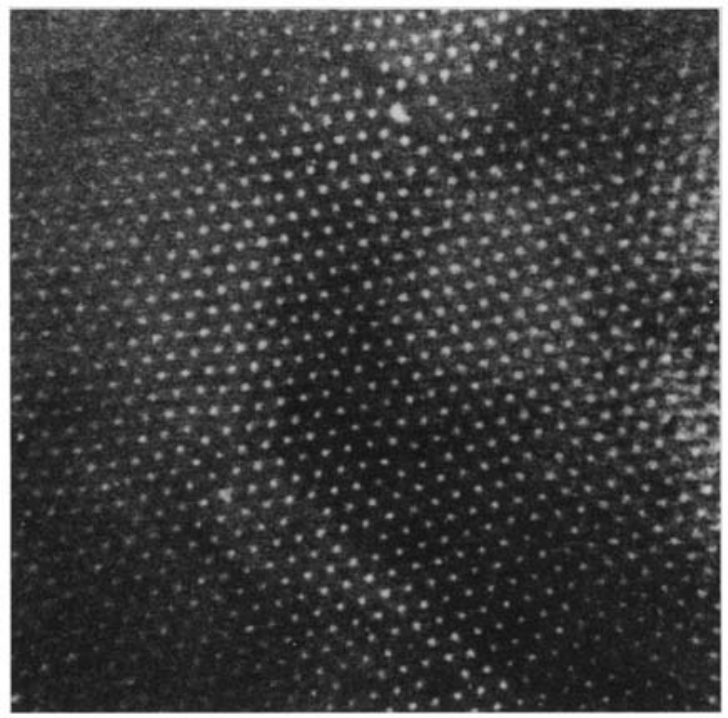

Figure 1. Micrograph of the first layer of the colloidal crystal near the glass surface. 


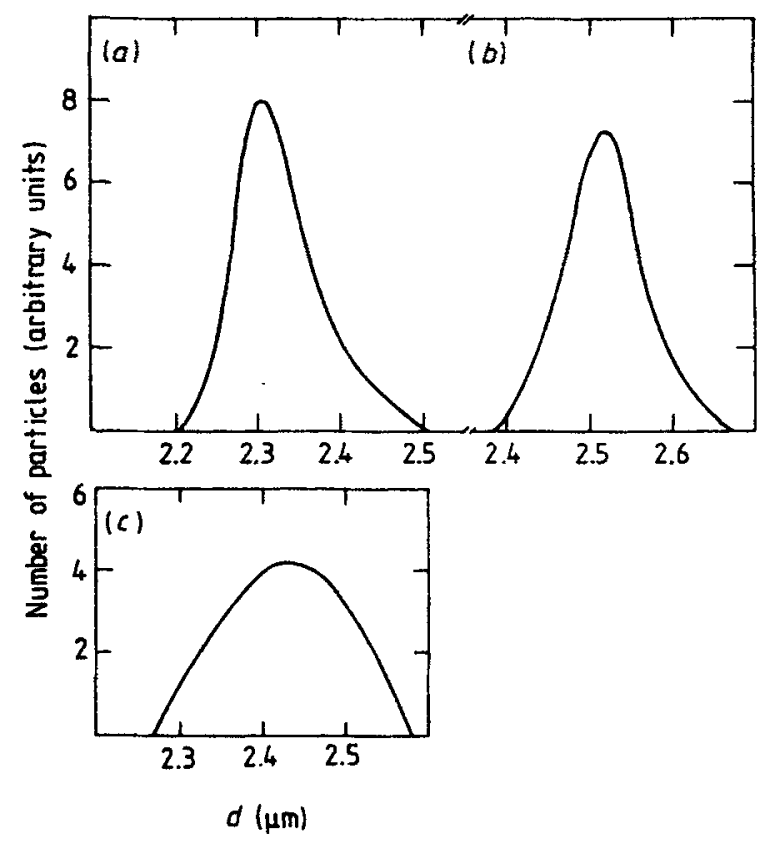

Figure 2. Distribution of $d$ in the first layer along [100] direction (a); along $(1, \sqrt{3}, 0)$ and $(1,-\sqrt{3}, 0)$ directions $(\mathbf{b})$; in the second layer along all directions $(\mathbf{c})$.

microscope intense light-heating, the interior layers disordered first and the first layer last. This is because the stabilizing potential provided by the glass surface decreases as the distance increases. In order to obtain the pair distribution function, $g(r)$, the particle positions in the first layer (figure 1) were loaded in the computer using the digitizer (Kesavamoorthy et al 1991). $g$ (r) was computed as

$$
N(r) / 2 \pi r \Delta r \rho,
$$

where $N(r)$ was the number of particles in an annular disc of radii $r$ and $r+\Delta r$ drawn with a particle at the centre, $\rho$ the number of particles per $\mathrm{cm}^{2} \cdot g(r)$ was averaged over about 500 particles and given in figure 3a. Also, given in figure $3 \mathrm{~b}$ is the $g(r)$ for the fourth layer. The first layer $g(r)$ showed many peaks as compared to that of fourth layer as expected. The curve relating the height of the peaks in $g(r)$ to their positions was least square fitted to an exponential and an algebraic function. The fit to the algebraic function was better implying the 2-D characteristic of the first layer. For the fourth layer also, the algebraic fit was better than with exponential. Though the colloidal crystal in a quartz container was FCC, a few layers near the container wall showed 2-D characteristic due to the stabilizing potential by the wall due to induced image charge effect.

\subsection{Behaviour of aggregates}

The colloidal particles interact via screened Coulomb potential. It prevents the particles touching from one another. The particles were separated by about $2.5 \mu \mathrm{m}$ for a suspension of 0.73 vol. $\%$ as shown in figure 2 . Stable aggregates of $2,3,4$, or 


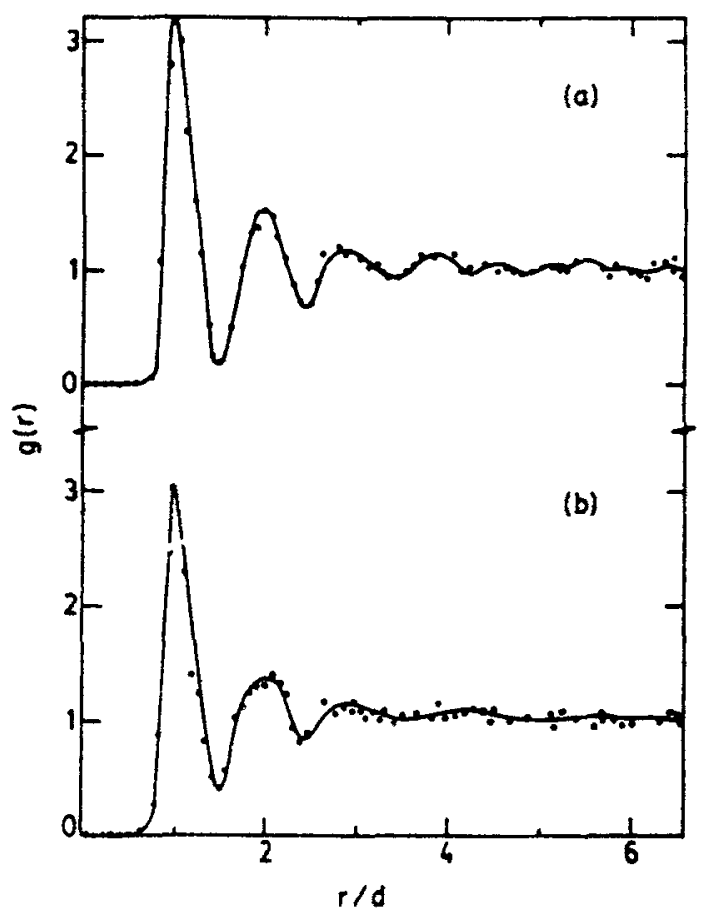

Figure 3. Pair distribution function, $g(r)$, of the first layer (a); fourth layer (b) of the colloidal crystal near the surface.

5 particles were observed in the colloidal crystal. The particles in an aggregate were vibrating continuously. The interparticle separation distance in an aggregate was not measurable using a microscope. The accuracy of measurement was poor so that the surface to surface particle separation of about a few tens of nanometer could not be measured. However, the particles in an aggregate were guessed to be separated by about a few tens of nanometer. Theoretical work is in progress to find a minimum in the potential at this separation distance. These aggregates were found as substitutional impurities in the colloidal crystal. They looked brighter than a single particle due to their coherent scattering of microscope light. The coordination number of a single particle in a layer of the crystal was peaked at six while that of an aggregate was higher than six. The surface to surface distance from an aggregate to the first neighbours were the same as that of a single particle to its neighbours. It is so because the surface charge density and the surface to surface separation, among the other parameters, decide the interaction strength. Since an aggregate is bigger than a particle, the coordination number is higher than six. The vibration of the particle was of $m$ sec period. The size of the image of a particle increased with the averaging time over which its scattered light was collected for imaging purpose (Kesavamoorthy et al 1989). The aggregate vibration was of larger period due to its heavy mass. Two particle aggregates performed a dumb-bell vibration. Three particle aggregates switched their configuration from the five o'clock orientation to the seven o'clock orientation. The six o'clock oriented aggregates were stable and did not switch over to any other orientation. In a fiveparticle-aggregate, four were forming a square in the plane of the layer and the fifth one 
was at the top of the square, lying above the plane. Occasionally the fifth particle switched over to the equivalent position to the other side of the plane. The four particles forming a square in the plane breath out so that enough space is created for the fifth particle to go from one side of plane to the other. Modelling of such aggregate vibrations is in progress.

\subsection{Flow under stress}

The sample cell was shaken and kept to rest on the sample platform of the microscope. Investigation was carried out on the first layer while the suspension flows under the effect of shear. The suspension near the glass surface flowed parallel to it. The particles were moving along with the suspension. As the flow velocity decreased, the particles in the first layer started jumping collectively in steps of particle separation distance. The defects like vacancies, dislocations, etc annealed as the flow velocity decreased further. The first layer particles, which constituted (111) plane of FCC before shearing, showed coexistence of square arrangements of particles in different grains during the flow under the shear. It is worthwhile to recall the observation that the grains of FCC structure with (111) plane and (100) plane parallel to the glass surface for suspension of vol. \% below 0.3 were coexisting under static conditions, while for higher volume fraction, only the grains of (111) planes existed. In these grains, the first plane of (111) grain (hexagonal arrangement of particles) and that of $(100)$ grain (square arrangement) were at the same distance from the glass surface while the second planar distance differed in accordance with the interplanar spacings of (111) and (100) planes. But, under shear, even for high volume fraction, (111) and (100) planes coexisted. The second layer in (100) grain was very close to the first layer. It was so close that the second layer particles appeared black under the microscope. It appeared black because the scattered light from this second layer interfered destructively with that from the first layer. Hence, these two layers would be within the depth of field which was of the order of $0.5 \mu \mathrm{m}$. (111) grains did not show such an effect. During the flow, the grain boundaries also moved along with the particles. When the flow stopped, the grains of (100) planes annealed back to (111) grains.

Another interesting aspect of flow was the dynamics of the particles near a slip boundary. Due to some pinning centres like agglomerates sticking to the glass surface, the first layer particles in some region did not flow while those in the nearby region flowed under stress. The size of the image of the particles in the static region increased monotonically as the slip boundary was approached while that of particles in the flowing region did not differ with respect to their distance from the boundary. As has been mentioned earlier, the size of the image constitute the area traversed by the particle in the time period over which its scattered light is collected for imaging. In our video recording system, each frame time is $40 \mathrm{~m} \mathrm{sec}$. Figure 4 shows the schematic of the particle size across the slip boundary. We understand this phenomena as arising due to the variation of bond length between the particle in the static region and that in the flowing region just across the slip boundary. As the particle in the flowing region moves forward, the bond length connecting it to a particle in the static region ahead of it decreases. This causes the static particle to move forward. When the moving particle comes nearest to the static particle, the latter is pushed away from the slip boundary. When a moving particle flows past the static particle, the bond length increases and the 


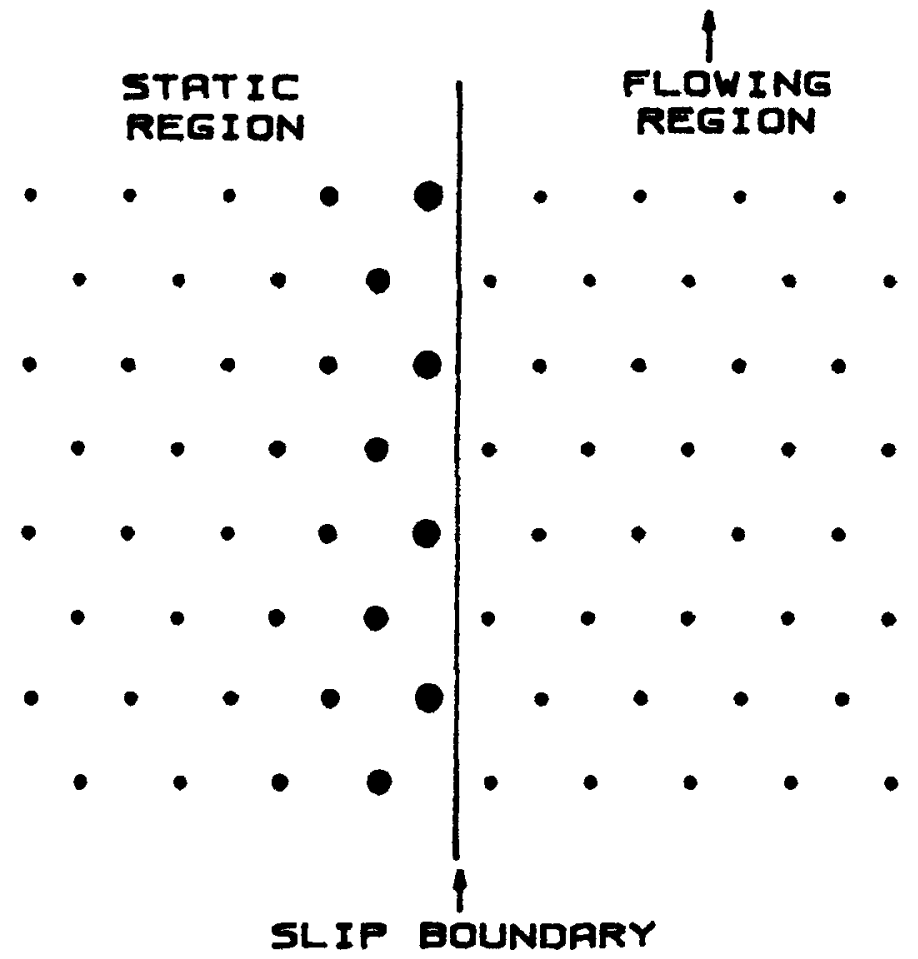

Figure 4. Schematic of the sizes of the images of the particles in the static and the flowing regions in the first layer.

static particle is pulled along the bond axis. Then, the interaction of the static particle with the moving particle weakens. But, the interaction of this static particle with the next moving particle coming from behind becomes predominant and the cycle of pushing and pulling of the static particle repeats. Hence, the static particle near the slip boundary traverses a wide area causing its image to be bigger. The effect of pushing and pulling by the flowing particle on the static particle decreases as the latter's distance from the boundary increases and hence its image size decreases. The flowing particle might take a course of wiggly movement along the flow direction due to the periodic variation in the bond length. Even then, its image size would not be bigger.

\section{Acknowledgement}

We acknowledge Dr Kanwar Krishan and Dr Baldev Raj for their encouragement.

\section{References}

Amstrong A J, Mocker R C and O'Sullivan W J 1989 J. Phys. : Condens. Matter 11707

Clark N A, Hurd A J and Ackerson B 1979 Natüre 28157

Hirtzel C S and Rajagopalan R 1985 Colloidal phenomena, advanced topics (Park Ridge, NY: Noyes)

Ise N 1986 Angew. Chem. Int. Ed. (English) 25323 
Ito K, Nakamura H, Yoshida H and Ise N 1988 J. Am. Chem. Soc. 1106955

Kesavamoorthy R 1990 J. Phys. A: Math. Gen. 24273

Kesavamoorthy R and Arora A K 1985 J. Phys. A: Math. Gen. 183389

Kesavamoorthy R, Sood A K. Tata B V R and Arora A K 1988 J. Phys. C: Solid State Phys. 214737

Kesavamoorthy R, Rajalakshmi M and Babu Rao C 1989 J. Phys.: Condens. Matter 17149

Kesavamoorthy R, Babu Rao C and Tata B V R 1991 J. Phys.: Condens. Matter 37973

Kesavamoorthy R, Shalabh Tandon, Xu S, Jagannathan S and Asher S A 1992 J. Colloid Int. Sci. 153188

Kesavamoorthy R, Babu Rao C and Baldev Raj 1993 J. Phys.. Condens. Matter 58805

Linsdsay H M and Chaikin P M 1982 J. Chem. Phys. 763774

Murray C A, Van Winkle D H and Wenk R A 1990 Phase Transitions 2193

Pansu B, Piranski P and Strzelecki L 1983 J. Phys. 44531

Piranski P 1980 Phys. Rev. Lett. 45569

Piranski P 1983 Contemp. Phys. 2425

Robbins M O, Kremer K and Grest G S 1988 J. Chem. Phys. 883286

Rundquist P A, Kesavamoorthy R, Jagannathan S and Asher S A 1991 J. Chem. Phys. $9 \$ 1249$

Russel W B, Saville D A and Schowatter W R 1988 Colloidal dispersions (Cambridge: Cambridge University Press) 